\title{
Non-equilibrium Structural Phase Transformations in Atomically Thin Transition Metal Dichalcogenides
}

Pawan Kumar ${ }^{1}$, James Horwath ${ }^{1}$, Alexandre Foucher ${ }^{1}$, Chrisopher Price ${ }^{1}$, Natalia Acero ${ }^{1}$, Vivek Shenoy $^{1}$, Deep Jariwala ${ }^{1}$, Eric Stach ${ }^{1}$ and Daan Hein Alsem ${ }^{2}$

${ }^{1}$ University of Pennsylvania, Philadelphia, Pennsylvania, United States, ${ }^{2}$ Hummingbird Scientific, Lacey, Washington, United States

Two-dimensional (2D) transition metal dichalcogenides (TMDCs) been the object of intense study due to their novel electronic and optical properties.[1] These materials can have a variety of structural forms. This is because the atoms can have different orientations within a single layer, or the individual layers can stack with different rotational variants.[2]

Here, we describe the strong effect that heating rates can have on the structural phase transformations that can occur in a specific TMDC, $\mathrm{MoS}_{2}$. We utilize a combination of aberration-corrected scanning transmission electron microscopy (JEOL NEOARM) and in-situ heating using microfabricated heating elements (Hummingbird Scientific) to directly visualize these processes and find that both the heating rate and final temperature can lead to very different structural phase transformations.

The use of a rapid heating rate $\left(25^{\circ} \mathrm{C} / \mathrm{sec}\right)$ to temperatures above $600{ }^{\circ} \mathrm{C}$ leads to the formation of highly ordered, crystalline hexagonal islands of sizes of less than $20 \mathrm{~nm}$ that are composed of a mixture of both the $2 \mathrm{H}$ and $3 \mathrm{R}$ phase (Figure 1). In contrast, the use of a slow heating rate $\left(25^{\circ} \mathrm{C} / \mathrm{min}\right)$ leads to the formation of ultra-nanocrystalline, sub-stoichiometric, and amorphous structures (Figure 2). The difference in the observed structural rearrangements can be explained by considering how the different rates of sulfur evaporation affect subsequent interaction with unbound molybdenum atoms on the surface. In the case of rapid heating, sulfur vacancies to form quickly, leading to S-adatoms on the MoS2 surface which quickly recombine with Mo adatoms that form at higher temperatures. However during slow heating the sulfur adatoms have time to recombine and form volatile sulfur compounds that leave the system prior to the temperatures at which Mo adatoms are present for subsequent recombination.

The ability to controllably create quantum-confined $\mathrm{MoS}_{2}$ islands leads to exciting new possibilities in engineering electronic structure in these systems and suggests novel device configurations in stacked twodimensional heterostructures.[3] 

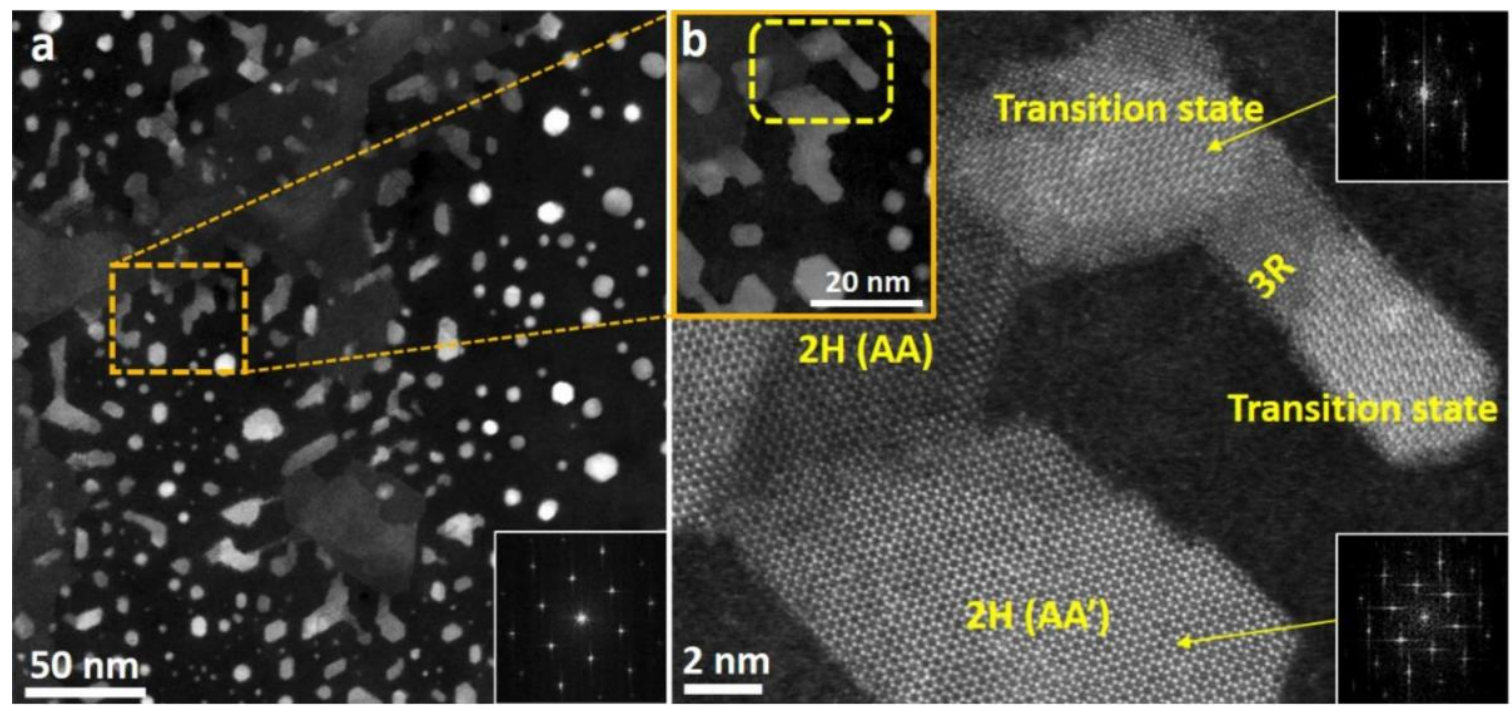

Figure 1. Rapid heating at $25^{\circ} \mathrm{C} / \mathrm{sec}$. (a) Low magnification ADF STEM image and inset selected area electron diffraction (SAED) pattern shows the high degree of crystallinity of disintegrated MoS2 from large 2D sheets, (b) Rotated, high magnification STEM image of highlighted boxed region in (a).
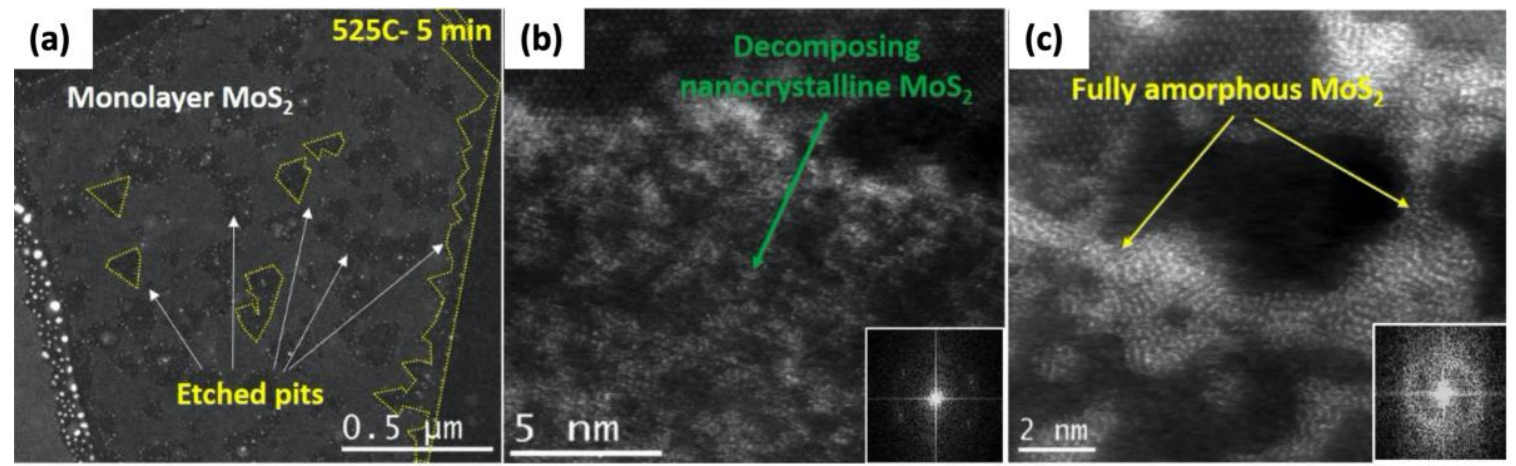

Figure 2. Slow heating at $25{ }^{\circ} \mathrm{C} / \mathrm{min}$. a) HAADF-STEM imaging of the MoS2 heated to $525{ }^{\circ} \mathrm{C}$ for 5 minutes. Triangular etch pits inside the monolayer along-with completely etched edges are evident. (b) HAADF-STEM image of few layer MoS2 heated to $550{ }^{\circ} \mathrm{C}$ for 5 minutes. Nanocrystalline order is evident in corresponding FFT which shows faint, diffused spots (inset) (c) Fully decomposed amorphous MoS2 with along with corresponding FFT inset $\left(550{ }^{\circ} \mathrm{C}\right.$ for $5-10$ mins $)$.

\section{References}

[1] Novoselov, K. S.; Jiang, D.; Schedin, F.; Booth, T. J.; Khotkevich, V. V.; Morozov, S. V.; Geim, A. K., Two-dimensional atomic crystals. Proceedings of the National Academy of Sciences of the United States of America 2005, 102 (30), 10451-10453.

[2] Lin, Y.-C.; Dumcenco, D. O.; Huang, Y.-S.; Suenaga, K., Atomic mechanism of the semiconductingto-metallic phase transition in single-layered MoS2. Nature Nanotechnology 2014, 9 (5), 391-396.

[3] Price, C. C.; Frey, N. C.; Jariwala, D.; Shenoy, V. B., Engineering Zero-Dimensional Quantum Confinement in Transition-Metal Dichalcogenide Heterostructures. ACS Nano 2019, 13 (7), 8303-8311. 